\title{
Reactivity of communities at equilibrium and periodic orbits
}

\author{
Frithjof Lutscher ${ }^{1}$ \\ Department of Mathematics and Statistics, Department of Biology, University of Ottawa, Ottawa, Canada \\ Xiaoying Wang $^{2}$ \\ Department of Mathematics and Statistics, University of Ottawa, Ottawa, Canada
}

\begin{abstract}
Reactivity measures the transient response of a system following a perturbation from a stable state. For steady states, the theory of reactivity is well developed and frequently applied. However, we find that reactivity depends critically on the scaling used in the equations. We therefore caution that calculations of reactivity from nondimensionalized models may be misleading. The attempt to extend reactivity theory to stable periodic orbits is very recent. We study reactivity of periodically forced and intrinsically generated periodic orbits. For periodically forced systems, we contribute a number of observations and examples that had previously received less attention. In particular, we systematically explore how reactivity depends on the timing of the perturbation. We then suggest ways to extend the theory to intrinsically generated periodic orbits. We investigate several possible global measures of reactivity of a periodic orbit and show that there likely is no single quantity to consistently measure the transient response of a system near a periodic orbit.
\end{abstract}

Keywords: reactivity, transient dynamics, periodic orbits

\section{Introduction}

When a system is perturbed, by a small amount, from an asymptotically stable equilibrium, asymptotic stability guarantees that the system returns to this equilibrium eventually. The resilience of a system is an asymptotic measure for how fast the system returns to this steady state (Pimm and

Email addresses: Frithjof.Lutscher@uottawa.ca (Frithjof Lutscher), xiaoyingwang@trentu.ca (Xiaoying Wang)

${ }^{1}$ Corresponding author.

${ }^{2}$ Permanent address: Department of Mathematics, Trent University, Peterborough, Ontario, Canada. 
Lawton, 1977). The study of transient dynamics is concerned with the possible states of the system between the initial perturbation and the asymptotic return to equilibrium (Neubert and Caswell 1997). For example, reactivity measures the response of a system immediately after a perturbation from the steady state. Loosely speaking, if all possible perturbations decay monotonically, we call the equilibrium non-reactive. If at least one perturbation initially grows before it eventually decays, we say that it amplifies and that the equilibrium is reactive. Characterizing reactivity of equilibrium states for continuous and discrete-time models is by now well understood and broadly applied; see e.g., Neubert and Caswell (1997); Caswell and Neubert (2005); Vesipa and Ridolfi (2017) and references therein.

However, many ecological systems are not at equilibrium. For example, seasonal forcing can appear to be at a stable equilibrium when looked at on the time scale of a year (like a stroboscopic image). When we ask how such periodically forced systems react to perturbations on small and intermediate time scales, we have to consider a temporal component in addition to the spatial component. Not only the magnitude and direction of the perturbation but also its timing within the periodic orbit may affect how strongly the systems responds to it. For example, during the winter, snowshoe hare is essentially the sole prey species available to predators such as lynx and owl in certain areas in northwestern Canada. During the summer, however, a number of other prey species are available (Krebs et al., 2001). It is therefore conceivable that the removal of a certain number of hares during the summer season would have a much smaller effect on the predator populations than the removal of the same number during the winter season.

The theory of transients in periodically forced systems is still in its infancy. Vesipa and Ridolfi (2017) defined reactivity for a seasonally forced system and investigated its properties and dependence on the interaction structure in the community. The authors also gave one example that illustrates that reactivity depends on the timing of the perturbation, but did not investigate this aspect in detail. This relationship between the timing of the perturbation and reactivity is the focus of our investigation here. Our work then allows us to answer questions of when during a cycle a system is particularly vulnerable or resilient to perturbations.

To set the stage, we begin by providing the background of the theory and give some examples for reactivity of steady states. In particular, we illustrate that reactivity may change under nondimensionalization, a previously overlooked or underappreciated fact. Then we define local reactivity 
for a forced periodic orbit and illustrate with an explicitly computable example that a periodic orbit can be locally reactive at some times and nonreactive at others. We then consider definitions of reactivity of a periodic orbit that would be intrinsic properties, independent of the timing of the perturbation. Somewhat surprisingly, we find that reactivity on the time scale of the periodic orbit

40 is not independent of the timing. Finally, we ask the question of how reactivity could be defined for intrinsically generated periodic orbits and show that some new phenomena arise.

\section{Background and terminology}

We begin by defining our terminology and notation. We consider a system of $k$ interactive species or compartments. We write $\mathbf{x}(t) \in \mathbb{R}^{k}$ for the state vector of the system at time $t$, where each component of the vector corresponds to the density of one of the species or compartments. The dynamics of the system are given by the equation

$$
\frac{\mathrm{d} \mathbf{x}(t)}{\mathrm{d} t}=\mathbf{F}(\mathbf{x}(t))
$$

For a given steady state, $\mathbf{x}^{*}$, we want to quantify the dynamics of small perturbations from that state, i.e., the behavior of solutions with initial condition $\mathbf{x}_{0}=\mathbf{x}^{*}+\mathbf{y}_{0}$, where $\left\|\mathbf{y}_{0}\right\|$ is small. Their behavior is determined by the solutions to the linearized equation

$$
\frac{\mathrm{d} \mathbf{y}(t)}{\mathrm{d} t}=\mathbf{A y}(t), \quad \mathbf{y}(0)=\mathbf{y}_{0},
$$

where $\mathbf{A}=D \mathbf{F}\left(\mathbf{x}^{*}\right)$ is the community matrix (the Jacobian) at the steady state. For any matrix $\mathbf{A}$, we denote by $\lambda_{1}(\mathbf{A})$ the eigenvalue with the largest real part. If this quantity is negative, i.e., $\operatorname{Re}\left(\lambda_{1}(\mathbf{A})\right)<0$, then all solutions of $(2)$ will eventually decay to zero exponentially with (at least) the rate $\operatorname{Re}\left(\lambda_{1}(\mathbf{A})\right)$ and $\mathbf{x}^{*}$ is asymptotically stable. Resilience is defined as the minimal return rate to steady state, i.e.,

$$
R:=-\operatorname{Re}\left(\lambda_{1}(\mathbf{A})\right)>0 .
$$

The larger $R$, the faster perturbations approach the steady state asymptotically for large times.

ॠThe inverse of resilience can be viewed as a measure of return time to steady state (Pimm and 45 Lawton, 1977).

Since resilience measures only the long-time decay rate of perturbations, Neubert and Caswell 
(1997) introduced several quantities to measure short- and intermediate-time aspects of perturbations. Reactivity measures the maximal instantaneous growth rate of a perturbation. Formally, it is defined as

$$
\sigma:=\max _{\left\|\mathbf{y}_{0}\right\| \neq 0}\left[\left.\left(\frac{1}{\|\mathbf{y}(t)\|} \frac{\mathrm{d}\|\mathbf{y}(t)\|}{\mathrm{d} t}\right)\right|_{t=0}\right]
$$

If $\sigma>0$ then at least one perturbation from the steady state will grow before it returns to the steady state. If $\sigma<0$ then all perturbations will decay to the steady state without growing initially. We call $\mathbf{x}^{*}$ reactive if it is locally stable and $\sigma>0$.

Observation 1: The definition of reactivity depends on the norm chosen, whereas resilience and stability do not. In particular, reactivity may depend on scaling. Hence, we have to use the dimensional (unscaled) model, not the nondimensional one to calculate the reactivity of a steady state. Previous authors, including the authors of this work, missed this point or at least did not comment on it (Neubert and Caswell, 1997; Wang and Lutscher, 2019). In Section 3.2, we give an example that demonstrates that a steady state can be reactive in the scaled model but not in the unscaled model and vice versa. In Section 3.3 , we give exact conditions for when the scaling of one variable in a linear two-dimensional system can change the reactivity.

It turns out to be particularly helpful to measure reactivity using the Euclidean distance, which results in the 2 -norm

$$
\|\mathbf{x}\|=\|\mathbf{x}\|_{2}=\left(x_{1}^{2}+x_{2}^{2}+\cdots+x_{n}^{2}\right)^{1 / 2} .
$$

With this norm, reactivity can be computed simply from the community matrix as (Neubert and Caswell, 1997)

$$
\sigma=\lambda_{1}(H(\mathbf{A})), \quad \text { with } \quad H(\mathbf{A})=\left(\mathbf{A}+\mathbf{A}^{\top}\right) / 2
$$

where $H(\mathbf{A})$ is the symmetric part of $\mathbf{A}, \mathbf{A}^{\top}$ is the transpose of $\mathbf{A}$, and $\lambda_{1}$ denotes the eigenvalue with the largest real part, as above. Since $H(\mathbf{A})$ is symmetric, all its eigenvalues are real, so that $\lambda_{1}(H(\mathbf{A}))$ is simply the largest eigenvalue.

To measure the behaviour of a perturbation at intermediate times, Neubert and Caswell (1997) define the amplification envelope

$$
\rho(t)=\max _{\left\|\mathbf{y}_{0}\right\| \neq 0} \frac{\|\mathbf{y}(t)\|}{\left\|\mathbf{y}_{0}\right\|}
$$

which measures, for any time $t>0$, the maximal deviation of any perturbation from the steady 

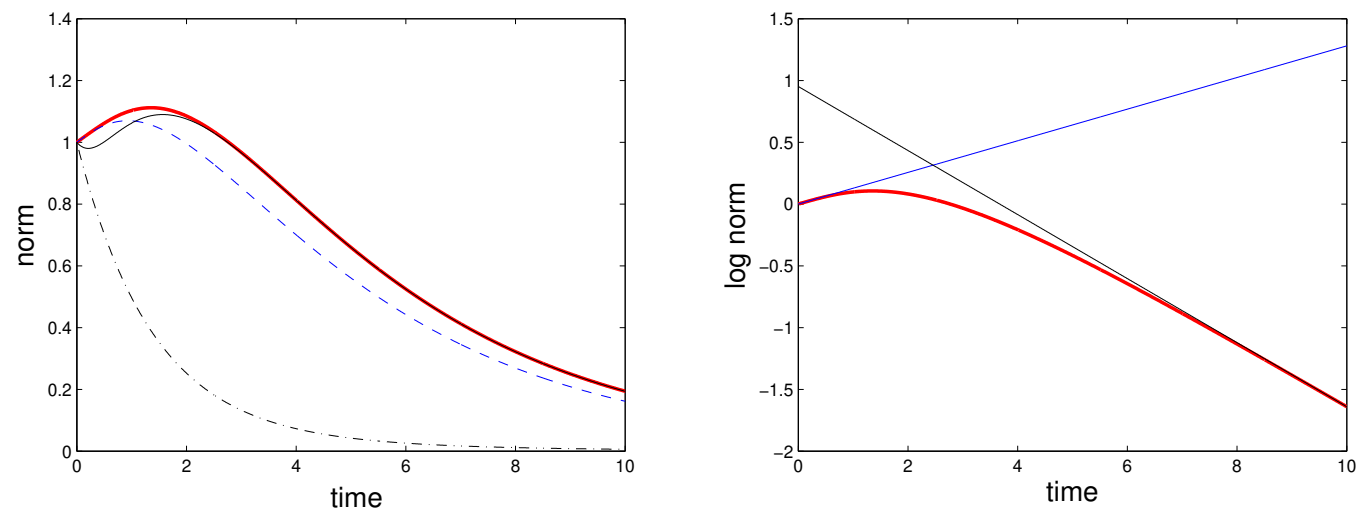

Figure 1: Illustration of resilience, reactivity, and amplification envelope. Left: The amplification envelope (red) is the maximum possible distance of a perturbation from the steady state. The distance of any particular perturbation may grow initially (blue, dashed), grow with some delay (black solid), or even decay (black, dash-dot). Right: The logarithm of the amplification envelope (red) with its slope at zero (reactivity, blue) and at infinity (negative resilience, black). The plots were produced from the example in Section 3.1 with parameter values $r=1, c=1$, $e=1, K=5$, and $m=0.2$. The perturbations of norm one were generated as $(\cos (\phi), \sin (\phi))^{\top}$ with $\phi=1.25$ (blue), $\phi=0.7$ (black solid), and $\phi=2.2$ (black dash-dot). The figure is inspired by figures 1a and 2 in Neubert and Caswell (1997).

state. Since the solution of $(2)$ is given by $\mathbf{y}(t)=\exp (\mathbf{A} t) \mathbf{y}(0)$, the amplification envelope can be calculated fairly easily with any standard software package as the matrix norm

$$
\rho(t)=\max _{\left\|\mathbf{y}_{0}\right\| \neq 0} \frac{\|\exp (\mathbf{A} t) \mathbf{y}(0)\|}{\left\|\mathbf{y}_{0}\right\|}=\|\exp (\mathbf{A} t)\| \|
$$

In general, there is not one initial perturbation that follows the amplification envelope all the time. Rather, for different $t$, different initial perturbations produce the maximum deviation. From the amplification envelope one can calculate the reactivity and resilience as the slope of $\ln (\rho(t))$ in the limits as $t \rightarrow 0$ and $t \rightarrow \infty$, namely

$$
\sigma=\frac{\mathrm{d}}{\mathrm{d} t} \ln (\rho(t))_{\mid t=0}, \quad R=-\frac{\mathrm{d}}{\mathrm{d} t} \ln (\rho(t))_{\mid t=\infty} .
$$

We illustrate all these quantities in Figure 1.

Observation 2: Scalar systems $(k=1)$ are not reactive.

When there is only a single species, we have the scalar equation

$$
\frac{\mathrm{d}}{\mathrm{d} t} x=F(x)
$$


The community "matrix" at a steady state $x^{*}$ is simply the derivative $F^{\prime}\left(x^{*}\right)$. If the steady state is asymptotically stable, then $F^{\prime}\left(x^{*}\right)<0$. The linearized equation

$$
\frac{\mathrm{d}}{\mathrm{d} t} y=F^{\prime}\left(x^{*}\right) y
$$

has the solution $y(t)=\exp \left(F^{\prime}\left(x^{*}\right) t\right) y_{0}$. Hence, if the steady state is stable, every solution of the linearized equation decays to zero monotonically. No state is reactive. Vesipa and Ridolfi (2017) give a very nice geometric illustration of conditions for reactivity in systems of two and more species and show that a steady state can only be reactive if the eigenvectors of the community matrix are not orthogonal.

\section{Reactivity for Equilibrium States}

We present some examples and illustrations for reactivity of stable equilibria. From the preceding observation, we know that the system must have at least two interacting species to show reactivity. We denote the community matrix for a system with two species at a locally asymptotically stable steady state by

$$
\mathbf{A}=\left[\begin{array}{ll}
a_{1} & a_{2} \\
a_{3} & a_{4}
\end{array}\right]
$$

Straightforward calculations show that the steady state is reactive exactly if

$$
4 a_{1} a_{4}<\left(a_{2}+a_{3}\right)^{2}
$$

see Appendix 7.1 for details. Since the right-hand side is nonnegative, the system will be reactive if the product $a_{1} a_{4}$ is negative. The entries $a_{1}$ and $a_{4}$ represent the impact that each species has on itself at steady state. If a species is self-limiting, then the corresponding entry is negative; if it is self-enhancing, the entry is positive. Hence, we have the following insight.

Observation 3: If one of the species is self-limiting at the steady state whereas the other is self-enhancing, then the system is reactive.

Predator-prey interactions often lead to reactive systems. Self limitation in the predator can result, for example, from a limit on the number of available territories (Turchin, 2003). Self enhancement in the prey can occur when the prey has an Allee effect (Murray, 2002). In this situation, 
the coexistence state is unconditionally reactive. Observation 3 also provides the link between reactivity and diffusion-driven instabilities or Turing patterns: the former is necessary for the latter (Neubert et al. 2002).

\subsection{Models of Lotka-Volterra type}

Many predator-prey models can be written in the generalized Lotka-Volterra form (Turchin. 2003)

$$
\frac{\mathrm{d} N}{\mathrm{~d} t}=f(N)-g(N) P, \quad \frac{\mathrm{d} P}{\mathrm{~d} t}=e g(N) P-m P,
$$

where $N$ is the prey density and $P$ the predator density. Furthermore, $f$ denotes the prey growth rate in the absence of the predator, $g$ stands for the functional response, $e$ is the conversion efficiency of the predator, and $m$ is the death rate of the predator. The community matrix at a positive steady state, $\left(N^{*}, P^{*}\right)$, is given by

$$
\mathbf{A}=\left[\begin{array}{cc}
f^{\prime}\left(N^{*}\right)-g^{\prime}\left(N^{*}\right) P^{*} & -m / e \\
e g^{\prime}\left(N^{*}\right) P^{*} & 0
\end{array}\right],
$$

in particular, $a_{4}=0$ in the notation above. Hence, by (11), the system is reactive as long as $a_{2}+a_{3} \neq 0$. This brings us to our next observation.

Observation 4: Generically, every asymptotically stable positive equilibrium point of 12 is reactive.

By "generically," we mean that for almost all choices of parameters, the steady state will be reactive. If it is not reactive for a given set of parameters, then the slightest change in one of the parameters will make the point reactive.

We illustrate the theory for model 12 with logistic growth and a linear functional response. We choose

$$
f(N)=r N(1-N / K) \quad \text { and } \quad g(N)=c N,
$$

where $r$ is the intrinsic growth rate of the prey, $K$ its carrying capacity, and $c$ the predation rate. The coexistence state of this model is given by

$$
N^{*}=\frac{m}{e c}, \quad P^{*}=\frac{r}{c}\left(1-\frac{m}{e c K}\right) .
$$


This point is globally asymptotically stable whenever it is biologically meaningful, i.e., when $P^{*}>0$ (Kot, 2001). The community matrix is

$$
\mathbf{A}=\left[\begin{array}{cc}
-N^{*} / K & -m / e \\
e c P^{*} & 0
\end{array}\right]
$$

The coexistence state is reactive unless $a_{2}+a_{3}=0$ or

$$
e r-\frac{m r}{c K}=\frac{m}{e}
$$

We illustrate this condition in Figure 2 .

\subsection{Other Types of Predator-Prey Models}

Predator-prey models that do not fall into the Lotka-Volterra framework can be nonreactive for large regions in parameter space. For the system to be nonreactive, the diagonal elements of the community matrix must have the same sign, i.e., either both species are self-enhancing at steady state or both are self-limiting. The prey is typically self-limiting at steady state. As mentioned above, self-limitation in the predator can result from a limit on the number of available territories (Turchin, 2003). One model that shows self-limitation of the predator is the Leslie model (Leslie, 1948)

$$
\dot{N}=r N(1-N / K)-c N P, \quad \dot{P}=s P(1-q P / N) .
$$

Parameters $r, c, K$ have the same meaning as above, $s$ denotes the intrinsic growth rate of the

predator, and $q$ is a scaling factor for the carrying capacity of the predator. The unique positive and asymptotically stable state is

$$
N^{*}=q P^{*}, \quad P^{*}=\frac{r K}{r q+c K}
$$

and the community matrix is

$$
\mathbf{A}=\left[\begin{array}{cc}
-N^{*} / K & -c N^{*} \\
s / q & -s
\end{array}\right]
$$


Both species are self-limiting at steady state. The steady state is reactive if

$$
4 \frac{s q r}{q r+c K}<\left(\frac{s}{q}-\frac{c r q K}{q r+c K}\right)^{2}
$$

The boundaries of this set with respect to parameters $s, K$ are depicted in Figure 2 .

To finish this section, we show that reactivity depends on the chosen scaling. There are many ways to nondimensionalize model (18). We choose $N=n K, P=p r / c$ and $t=\tau / r$ and write (18) in the equivalent nondimensional form

$$
\dot{n}=n(1-n)-n p, \quad \dot{p}=\tilde{s} p(1-\tilde{q} p / n)
$$

with $\tilde{s}=s / r$ and $\tilde{q}=q r /(c K)$, where $\dot{n}, \dot{p}$ now denote the derivatives with respect to $\tau$. The steady state is reactive if

$$
4 \frac{\tilde{s} \tilde{q}}{\tilde{q}+1}<\left(\frac{\tilde{s}}{\tilde{q}}-\frac{\tilde{q}}{\tilde{q}+1}\right)^{2}
$$

When we choose all dimensional parameters equal to unity, then also the dimensionless parameters equal unity, conditions (21) and (23) are not satisfied, and hence the positive steady states are not reactive. Changing the value of $K$ from 1 to 2 changes the value of $\tilde{q}$ to $1 / 2$. Consequently, (21) is not satisfied but (23) is. Hence, the steady state in the dimensional model is not reactive, but in the nondimensional model, it is. When we choose $K=1 / 2$ and $s=4$ (and all other parameters equal to unity), 21 is satisfied but (23) is not. Hence, the steady state in the dimensional model is reactive, but in the nondimensional model, it is not. In the next section, we study the question of how scaling affects reactivity in more detail with a linear two-dimensional model.

\subsection{Reactivity and Scaling}

We consider the two-dimensional linear system

$$
\dot{x}=a_{1} x+a_{2} y, \quad \dot{y}=a_{3} x+a_{4} y \text {. }
$$

We assume that the zero state is asymptotically stable, i.e., that $a_{1}+a_{4}<0$ and $a_{1} a_{4}-a_{2} a_{3}>0$. Then we introduce the scaled variable $z=c y$ for some $c>0$. In the new variables, the system reads

$$
\dot{x}=a_{1} x+a_{2} z / c, \quad \dot{z}=c a_{3} x+a_{4} z .
$$



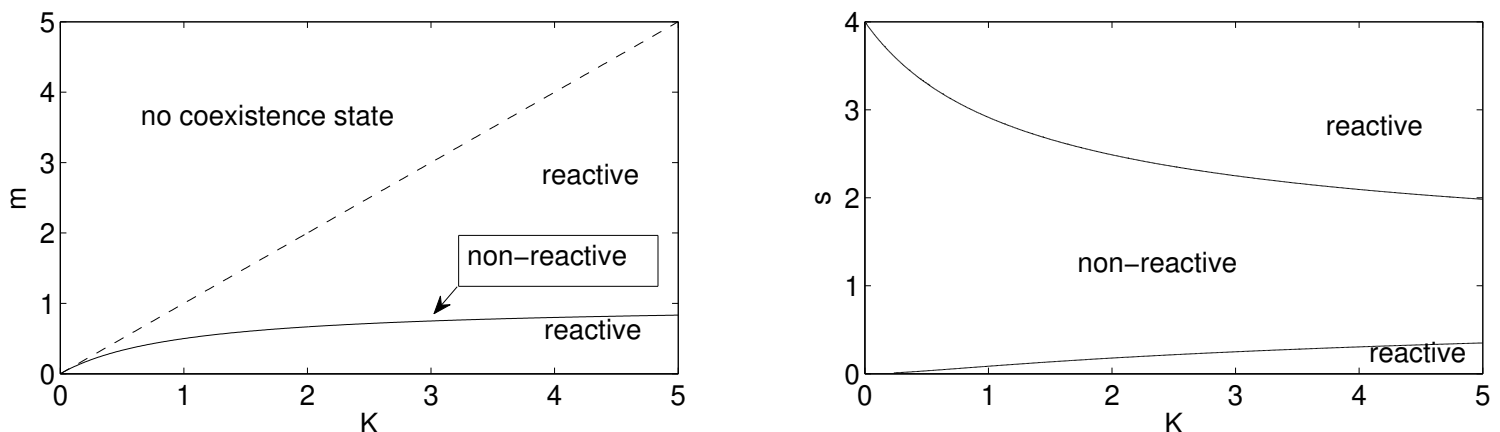

Figure 2: Illustrating the parameter ranges of reactivity for the Lotka-Volterra predator-prey model (left) and the Leslie model (right). Parameter values are $e=c=r=1$ in the Lotka-Volterra model and $r=c=q=1$ in the Leslie model.

The stability conditions are unaffected by the scaling. According to [11, the scaled system is reactive if

$$
4 a_{1} a_{4}<\left(\frac{a_{2}}{c}+c a_{3}\right)^{2}
$$

If $a_{1} a_{4}<0$, then the system is reactive independent of the scaling. If, however, $a_{1} a_{4}>0$, we can show that the system is reactive for very small and very large values of $c$, but not for some intermediate values. Clearly, 25) is satisfied in the limits as $c \rightarrow 0$ and $c \rightarrow \infty$. It remains to show that we can always find a range of $c$ where the inequality is violated. If $a_{2}$ and $a_{3}$ are of opposite sign, then setting $c=\sqrt{-a_{2} / a_{3}}>0$ makes the right-hand side of 25 equal to zero, so that the inequality is not satisfied. By continuity, there is an interval for $c$ where the inequality is violated. If $a_{2}$ and $a_{3}$ are both positive, then the expression inside the parentheses is concave up. The unique minimum occurs at $c=\sqrt{a_{2} / a_{3}}$ and is given by $2 \sqrt{a_{2} a_{3}}$. The inequality is satisfied at this minimum only if $a_{1} a_{4}<a_{2} a_{3}$. This, however, contradicts the stability condition $a_{1} a_{4}-a_{2} a_{3}>0$. The reasoning is similar when $a_{2}$ and $a_{3}$ are both negative. Hence, also in this case, there is an interval in $c$ where the 25 is not satisfied, which means that the system is not reactive. We summarize our findings as follows.

Observation 5: When $a_{1} a_{4}<0$, linear system (24) is reactive independent of the scaling $z=c y$. When $a_{1} a_{4}>0$ then the corresponding scaled system is reactive for large and small values of $c$ but not for some intermediate values.

115 In this simple example, we can determine the effects of scaling on the reactivity explicitly. In 
nonlinear models or models with more than two components, this task is much harder and more research needs to be done.

\section{Reactivity for periodically forced systems}

We begin our exploration of reactivity of periodic orbits with the case of periodically forced systems. Periodic forcing can describe annual variation in temperature and other abiotic variables that affect population and ecosystem dynamics. Our model equation now has the form

$$
\frac{\mathrm{d} \mathbf{x}(t)}{\mathrm{d} t}=\mathbf{F}(t, \mathbf{x}(t))
$$

where $\mathbf{F}$ is $T$-periodic in the first argument. We assume that this system has a locally stable, positive $T$-periodic orbit, which we denote by $\gamma(t)$. We pick a point on this periodic orbit, say $\gamma_{0}=\gamma\left(t_{0}\right)$, and a small perturbation, $\mathbf{y}_{0}$. Just as in the equilibrium case, we want to characterize the return of this perturbation to the periodic orbit, and just as before, we use linearization to obtain this characterization. The perturbation equation is now the time-dependent linear equation

$$
\frac{\mathrm{d} \mathbf{y}(t)}{\mathrm{d} t}=\mathbf{A}(t) \mathbf{y}(t), \quad \mathbf{y}\left(t_{0}\right)=\mathbf{y}_{0}
$$

where $\mathbf{A}(t)=D_{x} \mathbf{F}(t, \gamma(t))$. As before, reactivity is the maximum instantaneous growth rate from the initial perturbation, but unlike before, this quantity now depends on the point chosen along the periodic orbit. To indicate this dependence, we call it the local reactivity and define it as

$$
\sigma_{L}:=\sigma_{L}\left(t_{0}\right):=\max _{\left\|\mathbf{y}_{0}\right\| \neq 0}\left[\left.\left(\frac{1}{\|\mathbf{y}(t)\|} \frac{\mathrm{d}\|\mathbf{y}(t)\|}{\mathrm{d} t}\right)\right|_{t=t_{0}}\right]
$$

where $\mathbf{y}(t)$ is the solution of 27). This quantity can still be computed relatively easily from the formula

$$
\sigma_{L}\left(t_{0}\right)=\lambda_{1}\left(H\left(\mathbf{A}\left(t_{0}\right)\right)\right)
$$

which is just the analogous expression of (5).

Observation 6: The immediate response of a periodically forced system to a perturbation depends on the timing of the perturbation along the periodic orbit. 
We shall see in the examples below that the instantaneous reactivity may change sign over one period. Therefore, perturbations may have quite different effects, depending on where they occur along the periodic orbit. Vesipa and Ridolfi (2017) calculated amplification envelopes for four different times of perturbation in their predator-prey system and noted that there were clear differences, but they did not systematically study this dependency and they did not report a sign change in reactivity for their system.

We also define the local amplification envelope for $t>t_{0}$ as

$$
\rho_{L}\left(t, t_{0}\right)=\max _{\left\|\mathbf{y}_{0}\right\| \neq 0} \frac{\|\mathbf{y}(t)\|}{\left\|\mathbf{y}_{0}\right\|}
$$

We have the same relationship between $\sigma_{L}$ and $\rho_{L}$ as before, i.e.,

$$
\sigma_{L}=\sigma_{L}\left(t_{0}\right)=\frac{\mathrm{d}}{\mathrm{d} t} \ln \left(\rho_{L}\left(t, t_{0}\right)\right)_{\mid t=t_{0}}
$$

\subsection{Example: The periodically forced logistic equation}

We consider a single logistically growing population with time-periodic carrying capacity, i.e.,

$$
\frac{\mathrm{d} x(t)}{\mathrm{d} t}=r x(t)[1-x(t) / K(t)]
$$

where $K(t)$ is a positive $T$-periodic function. This equation has a unique positive globally stable $T$ periodic solution, $\gamma(t)$, which can be calculated analytically (see Appendix 7.2). The perturbation equation,

$$
\frac{\mathrm{d} y(t)}{\mathrm{d} t}=r[1-2 \gamma(t) / K(t)] y(t), \quad y\left(t_{0}\right)=y_{0},
$$

has the explicit solution

$$
y(t)=y\left(t_{0}\right) \exp \left(\int_{t_{0}}^{t} r[1-2 \gamma(s) / K(s)] \mathrm{d} s\right)
$$

Because of this simple expression, we can explicitly calculate the local amplification envelope as

$$
\rho_{L}\left(t, t_{0}\right)=\max _{\left|y_{0}\right| \neq 0} \frac{|y(t)|}{\left|y_{0}\right|}=\exp \left(\int_{t_{0}}^{t} r[1-2 \gamma(s) / K(s)] \mathrm{d} s\right)
$$



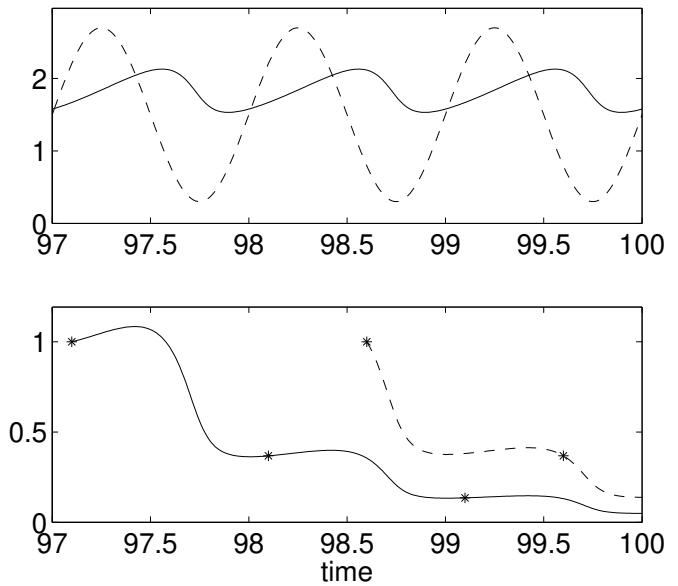

Figure 3: Top: Three periods of the periodic orbit $(\gamma(t)$, solid) of model $\sqrt{32}$ ) and half the carrying capacity $(K(t) / 2$, dashed). The periodic orbit is locally reactive where the dashed curve exceeds the solid curve; see (36). Bottom: The amplification envelopes (35) of two perturbations. The solid (dashed) curve represents a perturbation at a time where the orbit is (is not) locally reactive. The perturbation grows (decays) initially. The stars on the curves indicate how each perturbation decays over one period. Parameters are $r=1$ and $K(t)=3(1+0.8 \sin (2 \pi t))$.

and the local reactivity as

$$
\sigma_{L}\left(t_{0}\right)=\frac{\mathrm{d}}{\mathrm{d} t} \ln \left(\rho_{L}\left(t, t_{0}\right)\right)_{\mid t=t_{0}}=r\left[1-2 \gamma\left(t_{0}\right) / K\left(t_{0}\right)\right] .
$$

The local reactivity is positive where $\gamma\left(t_{0}\right)<K\left(t_{0}\right) / 2$ and negative where the opposite inequality

holds. In the former case, small perturbations will initially move further away from the periodic orbit; in the latter, they will initially approach the periodic orbit. Figure 3 shows the periodic orbit, the local reactivity, and two perturbations at different times, one where the orbit is locally reactive and one where it is not.

To obtain a measure of reactivity that is independent of the timing of the perturbation, we can define the maximal local reactivity

$$
\sigma_{M}=\max _{0 \leq t \leq T} \sigma_{L}(t)
$$

This maximum simply measures the largest possible instantaneous growth of a perturbation anywhere along the periodic orbit. Recall that the original definition of reactivity for an equilibrium also measures a maximum, namely over all possible directions of the perturbation. Hence, both approaches represent a worst case scenario. As such, if we define the reactivity of a periodic orbit 
as this maximum, $\sigma_{M}$, we remain within in the same spirit as the original definition of reactivity of an equilibrium point. This definition is independent of the time at which the perturbation occurs.

Such a definition of reactivity for a periodic orbit might be helpful in some cases, but could be an unnecessary overestimate of the effect of perturbations in others. For example, the plots in Figure 3 show that even though some perturbations grow initially, all decay on the time scale of the period of the system (indicated by the stars in the figure). It is tempting to think that the growth of a perturbation over the duration of one period could be independent of the timing of the perturbation since one integrates along the entire periodic orbit. We will show in the next section that this thought is incorrect.

\subsection{Period reactivity}

We choose the length of the periodic orbit as the inherent intermediate time scale of the system and study the continuous-time periodic system as a discrete-time system on this time scale via the period map (or stroboscopic map). Then we apply the existing definition of reactivity for discrete-time systems (Caswell and Neubert, 2005) to the period map.

We denote by $\mathbf{x}(t)$ a solution of (26), pick some initial time $t_{0}$, and consider the state of the system at discrete times, period $T$ apart, i.e., $\mathbf{z}_{n}=\mathbf{x}\left(n T+t_{0}\right)$ for $n=0,1,2, \ldots$ The sequence $\mathbf{z}_{0}, \mathbf{z}_{1}, \ldots$ solves the period map

$$
\mathbf{z}_{n+1}=\mathbf{P}\left(\mathbf{z}_{n}\right),
$$

where $\mathbf{z}_{n+1}$ is the solution of (26) at time $T$ with initial condition $\mathbf{x}(0)=\mathbf{z}_{n}$. A $T$-periodic orbit of system (26) corresponds to a fixed point $\mathbf{z}^{*}$ of $\mathbf{P}$. We note that the period map and the fixed point depend on $t_{0}$.

We linearize (38) at $\mathbf{z}^{*}$ and get the linear discrete equation

$$
\mathbf{w}_{n+1}=D \mathbf{P}\left(\mathbf{z}^{*}\right) \mathbf{w}_{n}=\mathbf{B}\left(\mathbf{z}^{*}\right) \mathbf{w}_{n}
$$

for some small initial perturbation $\mathbf{w}_{0}=\mathbf{z}^{*}-\mathbf{z}_{0}$. In general, the linearization will depend on $t_{0}$ as well.

We now define the period reactivity of the periodic orbit as the reactivity of the fixed point of this discrete system, i.e., the maximum rate of departure in the first iteration (Caswell and Neubert, 
2005). Formally, we have

$$
\sigma_{P}:=\ln \left(\max _{\left\|\mathbf{w}_{0}\right\| \neq 0} \frac{\left\|\mathbf{w}_{1}\right\|}{\left\|\mathbf{w}_{0}\right\|}\right)=\ln \left(\max _{\left\|\mathbf{w}_{0}\right\| \neq 0} \frac{\left\|\mathbf{B w}_{0}\right\|}{\left\|\mathbf{w}_{0}\right\|}\right)
$$

As in (5) for the equilibrium case, we can calculate the period reactivity in terms of the largest eigenvalue of a symmetric matrix obtained from B, namely its largest singular value, as (Caswell and Neubert, 2005)

$$
\sigma_{P}:=\sigma_{P}\left(t_{0}\right)=\ln \left(\sqrt{\lambda_{1}\left(\mathbf{B}^{T} \mathbf{B}\right)}\right) .
$$

The eigenvalues of $\mathbf{B}$ determine the stability of the periodic orbit: If all eigenvalues are inside the unit circle, the periodic orbit is locally asymptotically stable; if at least one eigenvalue is outside the circle, the orbit is unstable. While $\mathbf{B}$ may depend on $t_{0}$, the stability properties of the periodic orbit do not. The largest singular value, however, does in general depend on $t_{0}$, and with it the period reactivity. To understand this difference, we pick two different times, $t_{0}$ and $t_{1}$ along the periodic orbit as our base points. Then there is a nonsingular matrix $\mathbf{Q}$, such that the corresponding linearizations $\mathbf{B}\left(t_{0}\right)$ and $\mathbf{B}\left(t_{1}\right)$ are related as $\mathbf{B}\left(t_{0}\right)=\mathbf{Q}^{-1} \mathbf{B}\left(t_{1}\right) \mathbf{Q}$. In particular, $\mathbf{B}\left(t_{0}\right)$ and $\mathbf{B}\left(t_{1}\right)$ have the same eigenvalues, and the stability of the periodic orbit does not depend on the point chosen. The largest singular value, however, is not invariant under a similarity transform unless the transform is orthogonal, i.e., $\mathbf{Q}^{-1}=\mathbf{Q}^{\top}$.

Observation 7: In general, the period reactivity will depend on the timing of the perturbation since $\mathbf{B}$ does. For this reason, we explicitly noted this dependence in the definition.

\subsection{Example continued: The periodically forced logistic equation}

We continue the study of the periodically forced logistic growth equation from above to illustrate these ideas. From the explicit expression of the solution, we calculate the period map as (see Appendix 7.2

$$
z_{n+1}=\mathbf{P}\left(z_{n}\right)=\frac{z_{n} \mathrm{e}^{r T}}{1+z_{n} a\left(T+t_{0}, t_{0}\right)}, \quad a\left(T+t_{0}, t_{0}\right)=\int_{t_{0}}^{t_{0}+T} \frac{r \mathrm{e}^{r\left(s-t_{0}\right)}}{K(s)} \mathrm{d} s .
$$

The dependence of $a$ on $t_{0}$ indicates the choice of the point on the periodic orbit. The fixed point of $\mathbf{P}$ is

$$
z^{*}=\frac{\mathrm{e}^{r T}-1}{a\left(T+t_{0}, t_{0}\right)},
$$


The simple example of the forced logistic equation demonstrated that a periodic orbit may be reactive at some points and nonreactive at others, but on the level of one period, it was nonreactive. It turns out that the latter observation holds for all single-species, periodically forced equations.

which again depends on the choice of $t_{0}$. The linearization of $\mathbf{P}$ at $z^{*}$ equals $\mathbf{P}^{\prime}\left(z^{*}\right)=\mathrm{e}^{-r T}$, which is independent of $t_{0}$ in this particular case. Hence, the period reactivity of the periodic orbit is $\sigma_{P}=-r T<0$. In other words, on the time scale of the period, the periodic orbit is not reactive. In Figure 3 , the stars on the curves for the two perturbations in the bottom panel indicate that the perturbations decrease from one period to the next, even if they may increase in between.

Observation 8: A stable periodic orbit of a periodically forced single-species equation cannot be period reactive.

The reason behind Observation 6 is essentially the same as for Observation 2: in a one-dimensional system, the linearization is only a single number, not a matrix. Hence, the "singular value" is the same as the "eigenvalue." More formally, let the system be $\dot{x}=F(t, x)$ with $F(t+T, x)=F(t, x)$. Denote the $T$-periodic orbit by $\gamma(t)$. The linearized equation is

$$
\dot{y}=D_{x} F(t, \gamma(t)) y \text {. }
$$

Since $F$ is one-dimensional, we can solve explicitly for one period to get

$$
y(T)=y(0) \exp \left(\int_{0}^{T} D_{x} F(t, \gamma(t)) \mathrm{d} t\right)
$$

If the periodic orbit is stable, as we always assume, then the exponential term is less than unity. Hence, over the course of one period, the ratio $y(T) / y(0)$ has to decrease. As we shall see in the next section, Observation 6 does not apply to systems of two or more species.

\subsection{Computing period reactivity}

The preceding example is one of the rare cases where the period map and its linearization can be calculated explicitly. In general, we have to rely on numerical computations. But now we are faced with a problem. The period map that we are interested in can only be obtained by 
numerical integration. How could we possibly find the linearization of this numerically obtained map? Fortunately, there is an alternative method of calculating the linearization of the period map that does not require calculating the period map first. Rather, we have already linearized the vector field $\mathbf{F}$ in 26 at the periodic orbit, and we obtained the linearized equation (27). When we compute a fundamental solution of the linearized equation $(27)$ for one period, we obtain the so-called monodromy matrix. This matrix turns out to be precisely the linearization, $\mathbf{B}$, of $\mathbf{P}$ that we are looking for; see e.g. Perko (2000).

Hence, given a system of the form $(26)$, we first compute the $T$-periodic orbit, $\gamma(t)$. Since $\gamma$ must be locally stable, we can obtain the orbit by solving $(26)$ for large enough times. Next, we solve the matrix-valued differential equation

$$
\frac{\mathrm{d}}{\mathrm{d} t} \mathbf{X}=\mathbf{A}(t) \mathbf{X}, \quad \mathbf{X}\left(t_{0}\right)=\mathbf{I}_{k \times k},
$$

where $\mathbf{A}$ is as in 27) and the initial condition is the identity matrix. Then $\mathbf{B}\left(t_{0}\right)=\mathbf{X}(T)$, to which we then apply 40 .

We apply this methodology to a periodically forced Lotka-Volterra system and show that this system can be period reactive. We also show that there does not seem to be a simple relationship between the local and period reactivity. The system is given by

$$
\frac{\mathrm{d} N}{\mathrm{~d} t}=r N[1-N / K(t)]-c N P, \quad \frac{\mathrm{d} P}{\mathrm{~d} t}=e c N P-m P
$$

with a 1-periodic sinusoidally varying carrying capacity for the prey, i.e.,

$$
K(t)=K_{m}+K_{a} \sin (2 \pi t) .
$$

All parameters in the model are positive, except possibly $K_{a}$, which satisfies $\left|K_{a}\right|<K_{m}$, so that $K(t)>0$ for all $t$.

In the absence of the predator, this system is exactly the forced logistic equation that we studied in Section 4.1. Hence, there is a positive periodic orbit of the form $\bar{\gamma}(t)=(\bar{N}(t), 0)^{\top}$ that can be computed explicitly and that is globally stable within the set where $P=0$. The predator can persist in the system if its net growth rate for one period along the prey-only periodic orbit exceeds 

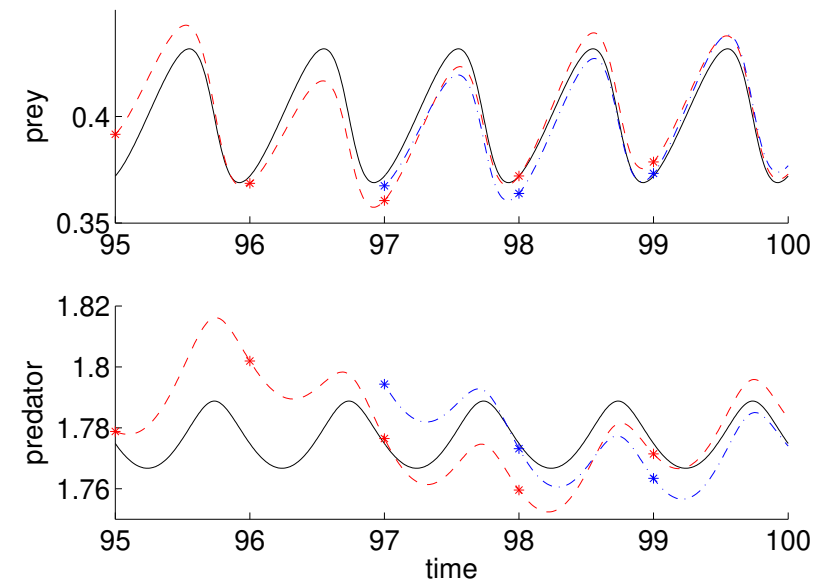

Figure 4: The periodic orbit (black) and two perturbations (red, amplifying; blue, non-amplifying) for the periodically forced Lotka-Volterra model 43 . Parameters are $r=5, c=2.5, e=0.5, m=0.5, K_{m}=5$, and $K_{a}=3.5$.

unity, i.e., if

$$
\frac{e c}{T} \int_{0}^{T} \bar{N}(t) \mathrm{d} t>m
$$

We are not aware of any method to explicitly obtain an analytic expression of a positive $T$-periodic orbit of predator and prey, but simple numerical integration with a standard software package (Matlab in our case), reveals a 1-periodic orbit, $\gamma(t)=\left(N^{*}(t), P^{*}(t)\right)^{\top}$, depicted as the black curves in Figure 4 . The figure also shows the trajectories of two different perturbations. The one in blue starts at $T=97$ and does not show amplification over one period, i.e., the Euclidean distance of the perturbation to the periodic orbit decreases from one period to the next. The one in red starts at $T=95$. It is chosen to produce the maximum amplification over one period. Initially, both perturbations have the same Euclidean distance from the periodic orbit.

To continue the computations, we find the linearization at the orbit as

$$
\mathbf{A}(t)=\left[\begin{array}{cc}
r-2 r N^{*}(t) / K(t)-c P^{*}(t) & -c N^{*}(t) \\
e c P^{*}(t) & e c N^{*}(t)-m
\end{array}\right]
$$

The local reactivity is computed easily from 29 . For the period reactivity, we solve 42 for each initial time $t \in[0, T]$. The results are plotted in Figure 5 for a range of parameter values. We see several different scenarios. In particular, there does not seem to be a clear relationship between the 
signs of $\sigma_{L}$ and $\sigma_{P}$. While the local reactivity is positive in (a)-(c), the period reactivity is positive in (a), changes sign in (b) and is negative in (c). In (d), the local reactivity changes sign while the period reactivity is positive. However, there appears to be a relationship between the peaks and troughs of the two measures of reactivity in that the peaks and troughs of the period reactivity seem to precede those of the local reactivity. 

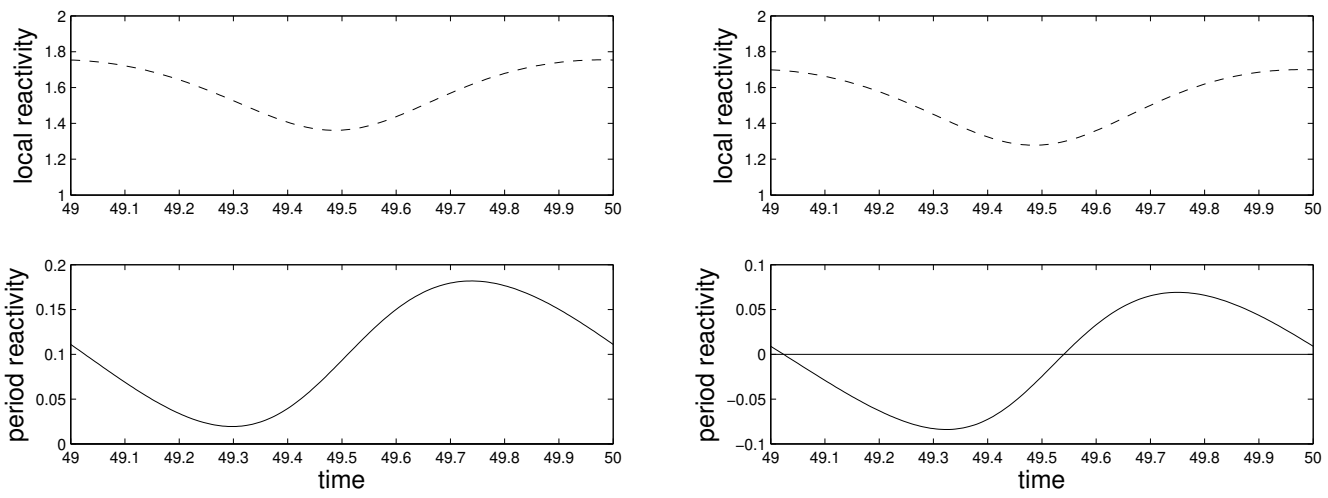

(a) $e=0.5, m=0.5$

(b) $e=0.45, m=0.5$
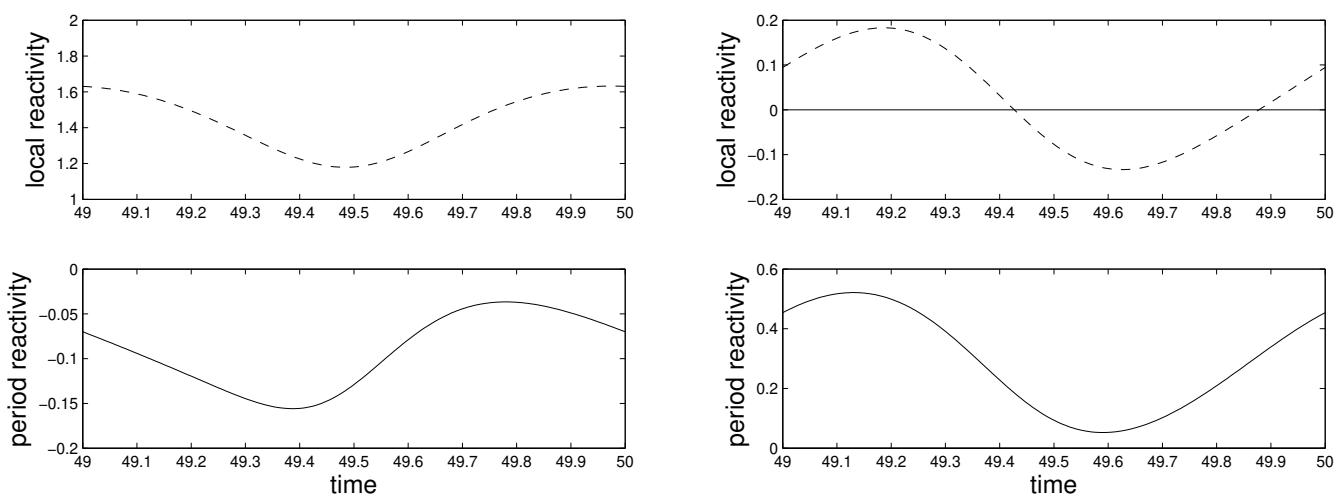

(c) $e=0.4, m=0.5$

(d) $e=0.2, m=1$

Figure 5: Four scenarios of reactivity in the periodically forced Lotka-Volterra predator-prey system 433. Each scenario shows the local (dashed, upper plot) and period (solid, lower plot) reactivity. In (a), local and period reactivity are positive for the entire orbit. In (b), the local reactivity is positive while the period reactivity changes sign. In (c),the local reactivity is positive while the period reactivity is negative. In (d), the period reactivity is positive while the local reactivity changes sign. Parameters are as indicated. Other parameters are $r=5, c=2.5$, $K_{m}=3$, and $K_{a}=1$. 


\section{Defining reactivity for intrinsically generated periodic orbits}

Predator-prey interactions are not only typical examples where a steady state can be reactive, they also often generate instabilities of the steady state, which can generate periodic orbits intrinsically. A typical example is the Rosenzweig-McArthur model (Kot, 2001), see below. We present some observations for how one might define reactivity for such intrinsically generated periodic orbits. It turns out that some concepts carry over directly from forced orbits, but others require more fundamental considerations about the quantities that we are interested in.

Our model equation is the autonomous equation, i.e.,

$$
\frac{\mathrm{d} \mathbf{x}(t)}{\mathrm{d} t}=\mathbf{F}(\mathbf{x}(t))
$$

where $F: \mathbb{R}^{k} \rightarrow \mathbb{R}^{k}$. We assume that this system has a locally stable, positive $T$-periodic orbit, which we denote by $\gamma(t)$ and we pick a point $\gamma_{0}=\gamma\left(t_{0}\right)$ on this orbit. Also as before, the linearization at this periodic orbit is given by

$$
\frac{\mathrm{d} \mathbf{y}(t)}{\mathrm{d} t}=\mathbf{A}(t) \mathbf{y}(t), \quad \mathbf{y}\left(t_{0}\right)=\mathbf{y}_{0},
$$

where $\mathbf{A}(t)=D_{x} \mathbf{F}(\gamma(t))$. With this notation, the local reactivity, $\sigma_{L}\left(\gamma_{0}\right)$, of $\gamma(t)$ can be defined exactly as before in 28 and calculated as in 29 .

\subsection{The Rosenzweig-MacArthur model}

We consider the well-known Rosenzweig-MacArthur model, which is a special form of 12 with logistic growth of the prey and a Holling type-II functional response. The model reads

$$
\dot{N}=r N(1-N / K)-\frac{c_{1} N P}{1+a_{1} N}, \quad \dot{P}=\frac{e_{1} c_{1} N P}{1+a_{1} N}-m_{1} P
$$

where $r$ and $K$ are the growth rate and carrying capacity in the logistic growth of the prey, $c_{1}$ is the maximum predation rate, $a_{1}$ encompasses the handling time, $e_{1}$ is the conversion efficiency, and $m_{1}$ is the death rate of the predator. The predator can persist in the system if $c_{1} e_{1}>m_{1}$ and $K$ is large enough. For increasing $K$, the coexistence state eventually becomes unstable in a Hopf bifurcation where a unique, globally stable limit cycle arises (Kot, 2001). Figure 6 illustrates this 

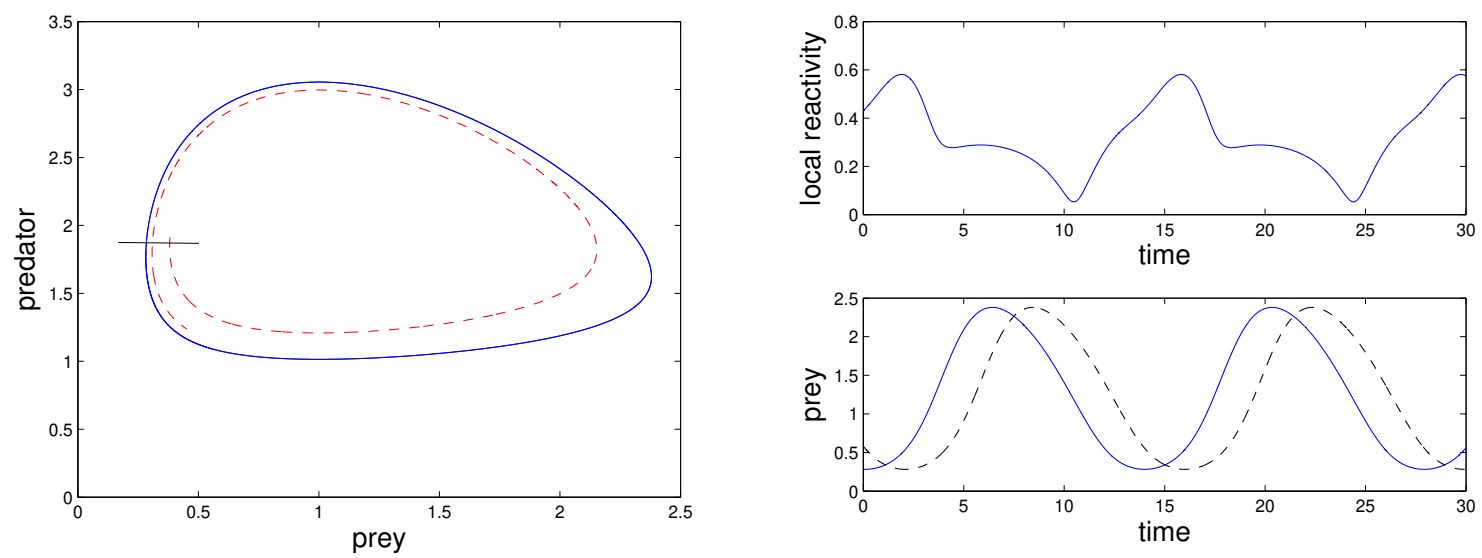

Figure 6: Left plot: The unique, stable periodic orbit of the Rosenzweig-MacArthur system 48. Right top: Local reactivity of the periodic orbit according to definition 28 and matrix 49 . Right bottom: illustration of a phase shift arising from a perturbation; see text for details. Parameters are $r=1.5, K=3.5, m_{1}=0.5, c_{1}=1, e_{1}=1$, and $a_{1}=1$.

limit cycle in the phase plane. The linearization of 480 is given by

$$
\mathbf{A}(t)=\left[\begin{array}{cc}
r-\frac{2 r N(t)}{K}-\frac{c_{1} P(t)}{\left(1+a_{1} N(t)\right)^{2}} & -\frac{c_{1} N(t)}{\left(1+a_{1} N(t)\right)^{-1}} \\
\frac{e_{1} c_{1} P(t)}{\left(1+a_{1} N(t)\right)^{2}} & \frac{e_{1} c_{1} N(t)}{\left(1+a_{1} N(t)\right)^{1}}-m_{1}
\end{array}\right]
$$

We calculate the local reactivity from this matrix according to (28). For the parameters chosen in Figure 6, this quantity is positive throughout the periodic orbit. Hence, a local perturbation can initially grow, no matter where along the periodic orbit it occurs.

When we want to measure reactivity on the time scale of the periodic orbits, things start to differ from the case of periodically forced orbits. We can, of course, still define the period map $\mathbf{P}: \mathbb{R}^{k} \rightarrow \mathbb{R}^{k}$, as in 38 , and its linearization (39). However, this map is only partially helpful in characterizing how solutions from perturbations approach the periodic orbit, as the following observation shows.

Observation 9: The period reactivity (40) of a periodic orbit in the autonomous system 446 is never negative.

It is a standard result in dynamical systems theory that the period map of periodic orbit in an autonomous system has an eigenvalue equal to unity with $\gamma^{\prime}\left(t_{0}\right)$ as the corresponding eigenvector 
(Perko, 2000). Hence, we have $\mathbf{B} \gamma^{\prime}\left(t_{0}\right)=\gamma^{\prime}\left(t_{0}\right)$. In particular, the maximum in 40) is bounded below by unity, so that $\sigma_{P} \geq 0$.

A more biological explanation of this results is as follows. If a small perturbation of $\gamma\left(t_{0}\right)$ at time $t_{0}$ brings the system to some state that happens to be on the periodic orbit as well, say $\gamma\left(t_{1}\right)$, then after one period, we have $\gamma\left(t_{i}+T\right)=\gamma\left(t_{i}\right)$. Hence, after one period, the system is back at exactly the same state as it was at the time of the perturbation, and therefore the distance between the points is unchanged. As a result, the original system and the perturbed system will always trail one another but their distance will not converge to zero, even though the periodic orbit is assumed to be locally stable. The perturbation will simply lead to a shift in the phase of the periodic orbit (see Figure 6). This situation may sound contrived, but in some cases, we might be interested in such a phase shift. For example, if a system shifts phase, the timing of interactions with other species may change.

\subsection{Which distance should we measure?}

The preceding observation forces us to reconsider the very definition of reactivity and amplification envelope and, hence, ultimately what we mean by distance. Are we interested in how far the solution after a perturbation can be from the solution without the perturbation at any given time? Or are we interested in how far the solution after a perturbation can be from a certain attractive invariant set? If the perturbation is from a steady state, then the two distance measures are identical since the attractive invariant set is the steady state itself. To formulate these two possibilities more mathematically, we denote the $T$-periodic orbit of system 46 as $\gamma(t)$, the point at which the perturbation occurs as $\gamma_{0}=\gamma\left(t_{0}\right)$, the (small) perturbation by $\mathbf{y}_{0}$, and the solution of (46) with initial condition $\gamma_{0}+\mathbf{y}_{0}$ by $\mathbf{x}_{1}(t)$.

The maximal distance between the solutions of the perturbed and unperturbed system at time $t$ is the maximal "point distance"

$$
\max _{\left\|\mathbf{y}_{0}\right\| \neq 0} \frac{\operatorname{dist}\left(x_{1}(t), \gamma\left(t_{0}+t\right)\right)}{\left\|\mathbf{y}_{0}\right\|}=\max _{\left\|\mathbf{y}_{0}\right\| \neq 0} \frac{\left\|x_{1}(t)-\gamma\left(t_{0}+t\right)\right\|}{\left\|\mathbf{y}_{0}\right\|}
$$

If $\left\|\mathbf{y}_{0}\right\|$ is small enough, then the linearized equation (47) provides a good approximation to this quantity. In particular, this is the distance measure underlying the definition of local reactivity and, for $t=T$, also for period reactivity as studied above. We have seen that this measure detects 
phase shifts. The distance between the perturbed and unperturbed solutions in this sense will in general not converge to zero, even though the periodic orbit is assumed to be locally stable.

Alternatively, the maximal distance between the perturbed solution at time $t$ and the periodic orbit is the maximal "set distance"

$$
\max _{\left\|\mathbf{y}_{0}\right\| \neq 0} \frac{\operatorname{dist}\left(x_{1}(t), \gamma\right)}{\left\|\mathbf{y}_{0}\right\|}=\max _{\left\|\mathbf{y}_{0}\right\| \neq 0} \frac{\min _{\tau \in[0, T]}\left(x_{1}(t), \gamma\left(t_{0}+\tau\right)\right)}{\left\|\mathbf{y}_{0}\right\|}
$$

Observation 10: The restricted period reactivity of a planar periodic orbit in the autonomous system 46 is never positive.

The reason behind this observation is again a dimensional argument as for Observations 2 and 7 . 
The Poincaré map of a periodic orbit in a planar system is one dimensional. Because we assume the orbit to be stable, this map has to be contracting, i.e., $|\overline{\mathbf{B}}|<1$. Since $\overline{\mathbf{B}}$ is a single number (and not a matrix), the expression inside the logarithm in 40 is less than unity. An illustration of the geometric argument behind this observation is given in Figure 6. The dashed curve corresponds to a solution of the perturbed system, the black line segment indicates a hyperplane. Since the solution curve has to approach the periodic orbit, and since the system is planar (and no intersections can arise), the intersections of the solution with the black line segment must be monotone; see also the proof of the famous Poincaré-Bendixon theorem (Perko, 2000).

The difficulty in defining reactivity of a point on a periodic orbit through the Poincaré map is that this map depends on the choice of the hyperplane. Different choices of hyperplanes lead to different maps (essentially different coordinate systems). These differences do not affect whether eigenvalues of $\overline{\mathbf{B}}$ are within or outside the periodic orbit, i.e., $\overline{\mathbf{B}}$ can be used to determine stability of the orbit. However, as we saw in Observation 1, reactivity does depend on the chosen coordinate system. In the planar example above, this issue does not arise because we have only one dimension.

\subsection{An example with three species}

The question then becomes whether a periodic orbit in higher space dimensions can be period reactive in the restricted sense, and what minimum dimension might be necessary. We give an example of a three-species food chain that shows period reactivity in the restricted sense. The equations for the prey $(N)$, the primary consumer $(P)$, and the top predator $(Q)$ are

$$
\dot{N}=r N(1-N / K)-c_{1} N P, \quad \dot{P}=e_{1} c_{1} N P-m_{1} P-\frac{c_{2} P Q}{1+a_{2} P}, \quad \dot{Q}=\frac{e_{2} c_{2} P Q}{1+a_{2} P}-m_{2} Q
$$

Our aim is not to give a complete description of the dynamics of this model. In fact, it is known that three-species food chain models can exhibit chaotic behavior (Hastings and Powell, 1991). Instead, we find parameter values for which there is a stable periodic orbit and pick a point $(\bar{N}, \bar{P}, \bar{Q})$ on this orbit. We consider perturbations with Euclidean distance 0.1 from this point. We use spherical coordinates, pick random angles $\phi$ and $\theta$, and add the perturbation $0.1 *(\sin \theta \cos \phi, \sin \theta \sin \phi, \cos \theta)$ to the point. Then we plot (i) how the distance (50) of the solution starting at this perturbation to the solution starting at the periodic orbit changes over time (blue curves) and (ii) how the distance (51) of the perturbation to the periodic orbit changes over time (black curves). The top panel in Figure 7 shows the periodic orbit. The middle and bottom panels show two solutions with different 
properties. In the middle panel, the point distance 50 increases initially (i.e., the orbit is locally reactive at this point), then decreases overall and settles at a (necessarily) periodic pattern that does not approach zero. We expect that a phase shift happened. The corresponding set distance 51 is initially already much lower than the point distance, indicating that, indeed, the perturbation is close to the periodic orbit, just not to the point on the orbit where the perturbation occurred. The set distance eventually decreases to zero as expected from the stability of the orbit (but not monotonically). In the bottom panel, the point distance initially decreases (i.e., the solution attenuates), but eventually grows from one period to the next and reaches a periodic pattern much higher than in the middle panel. The corresponding set distance is initially (almost) the same as the point distance, indicating that the perturbation occurred mostly perpendicular to the direction of the orbit. Sometime between time one and two, the set distance is larger than it is initially, indicating that there is some amplification occurring. The set distance eventually decreases as it should.

\section{Discussion}

Ever since its beginning, the theoretical study of ecological dynamics via dynamical systems has focused strongly on asymptotic analysis: stable steady states and periodic orbits. The occurrence of transients is sometimes mentioned in passing, for example to explain that simulations were run until transients had died out, but often not at all. The importance of transients has been emphasized much more strongly within the past two decades, but there is still no coherent theory on the topic. There are many different types of transient dynamics, for example those that result from stochastic events or those that result from changes in model parameters that induce bifurcations; see Hastings et al. (2018) for a recent review. The study of transient dynamics requires ideas and tools that are quite different from those for asymptotic dynamics.

The one aspect that we studied here is the response of a system after a small perturbation from an asymptotically stable state. In the case where this state is an equilibrium point in a system of differential equations, the theory goes back to the work by Neubert and Caswell (1997), who defined and studied reactivity and amplification envelope as alternative measures to resilience. ॠThey established a close connection between reactivity and Turing pattern formation Neubert et al. 2002), and they expanded the theory to cover discrete-time systems (Caswell and Neubert, 2005); see also Vesipa and Ridolfi (2017) for a more comprehensive review of the applications. 

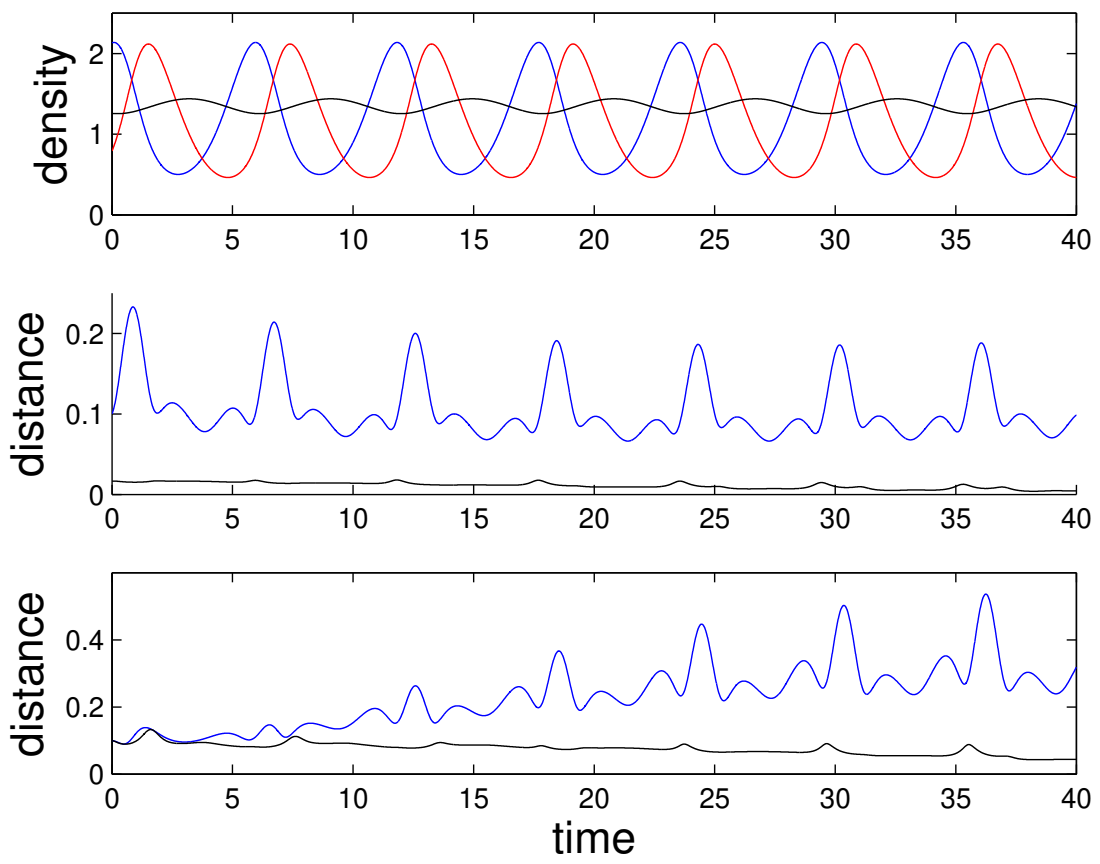

Figure 7: Top panel: A stable periodic orbit of the tri-trophic food chain model 52 with prey (blue), primary consumer (red) and top predator (black). Middle panel: The point distance (blue) and the set distance (black) of a perturbation at time $t_{0}=0$ when $\phi=4.4123$ and $\theta=1.3679$. Bottom panel: Same as middle panel with $\phi=6.0271$ and $\theta=2.4031$. Parameters are $r=1.5, K=5, m_{1}=0.5, e_{2}=0.4, m_{2}=0.2$, and $c_{1}=e_{1}=c_{2}=a_{2}=1$. 
Our first observation that reactivity may depend on scaling whereas asymptotic dynamics do not illustrates the differences between asymptotic and transient behavior on a fundamental level. While we could completely determine the effect of scaling on reactivity using the Euclidean norm in a twodimensional linear system, many open questions remain about higher dimensions and nonlinear systems.

The application of reactivity to periodic orbits is much more recent, in fact, we know only of the work by Vesipa and Ridolfi (2017) to address this question in the context of periodically forced systems. The motivation and usefulness of such an extension is obvious: many ecosystems are subject to annual forcing and are not at equilibrium.

Our work contributes a number of observations that, we hope, will advance the use and understanding of the concept of reactivity in this context. The major observation and subsequent result is that reactivity of a periodically forced orbit depends on the timing of the perturbation and can change sign along the orbit. Hence, a system may be highly sensitive to perturbations at some times while being insensitive at others. We also showed that the 'obvious solution' to this problem, namely to define reactivity of a periodic orbit on the level of the period is not a solution at all because the period reactivity also depends on timing. There seems to be no clear relation between the local and period reactivity. The situation is even more complicated for intrinsically generated periodic orbits. We showed that the period reactivity is never negative since perturbations can induce phase shifts, so that solutions do not converge to solutions on the periodic orbit pointwise but only in the sense of set distance. In some situations, one may want to know by how much a perturbed solution differs from the corresponding unperturbed solution, in particular when the timing of peaks and troughs of the populations is important. In other cases, one might only care whether the population densities will differ by much of what is normally seen without perturbation, but not about the timing of when that would happen. Our results show that it is still an open question how to define such a measure in general.

We also showed that the dimensionality of the system determines whether it can be reactive or not. Equilibrium points in scalar dynamics cannot be reactive but in more than one dimension, they can. Forced periodic orbits can be locally reactive in one dimension but require two dimensions to possibly be period reactive. Intrinsic periodic orbits cannot be period reactive in two dimensions but it likely can be in three dimensions (if one can agree on an appropriately extended definition of the concept). 
A particular class of forced systems are impulsive- or hybrid system, sometimes also referred

\section{Appendix}

7.1. The general reactivity condition (11)

The community matrix

$$
\mathbf{A}=\left[\begin{array}{ll}
a_{1} & a_{2} \\
a_{3} & a_{4}
\end{array}\right]
$$


is stable if and only if $\operatorname{tr}(\mathbf{A})=a_{1}+a_{4}<0$ and $\operatorname{det}(\mathbf{A})=a_{1} a_{4}-a_{2} a_{3}>0$. The symmetric part of $\mathbf{A}$ is

$$
H(\mathbf{A})=\left[\begin{array}{ll}
a_{1} & h \\
h & a_{4}
\end{array}\right], \quad h=\left(a_{2}+a_{3}\right) / 2 .
$$

Since $H(\mathbf{A})$ is symmetric, its eigenvalues are real. Since $\operatorname{tr}(\mathbf{A})=\operatorname{tr} H(\mathbf{A})$, we have $\operatorname{tr} H(\mathbf{A})<0$. For a positive eigenvalue of $H(\mathbf{A})$, we then require that the determinant be negative, i.e.

$$
a_{1} a_{4}<h^{2}
$$

Generically, we have $h \neq 0$, so that the condition is satisfied when $a_{1} a_{4} \leq 0$. In particular, it is

\subsection{The time-dependent logistic model}

In this appendix, we give all the detail calculations for solutions, stability, and reactivity of the logistic equation with time-periodic carrying capacity, i.e.,

$$
\frac{\mathrm{d} x(t)}{\mathrm{d} t}=r x(t)[1-x(t) / K(t)], \quad K(t+T)=K(t)
$$

where $r$ and $K$ are positive. The substitution $\tilde{x}(t)=1 / x(t)$ gives the linear inhomogeneous differential equation

$$
\frac{\mathrm{d} \tilde{x}(t)}{\mathrm{d} t}=-r \tilde{x}(t)+\frac{r}{K(t)}
$$

This equation can be solved by using integrating factors to get

$$
\tilde{x}(t)=\mathrm{e}^{-r\left(t-t_{0}\right)} \tilde{x}\left(t_{0}\right)+\int_{t_{0}}^{t} \frac{r \mathrm{e}^{-r(t-s)}}{K(s)} \mathrm{d} s .
$$

In the original variable, this solution reads

$$
x(t)=\frac{x\left(t_{0}\right) e^{r\left(t-t_{0}\right)}}{1+x\left(t_{0}\right) a\left(t, t_{0}\right)}, \quad \text { where } \quad a\left(t, t_{0}\right)=\int_{t_{0}}^{t} \frac{r \mathrm{e}^{r\left(s-t_{0}\right)}}{K(s)} \mathrm{d} s .
$$

In particular, we have

$$
x\left(T+t_{0}\right)=\frac{x\left(t_{0}\right) e^{r T}}{1+x\left(t_{0}\right) a\left(T+t_{0}, t_{0}\right)} .
$$


Hence, the Poincaré map is given by

$$
\mathbf{P}\left(z, t_{0}\right)=\frac{z e^{r T}}{1+z a\left(T+t_{0}, t_{0}\right)}
$$

This map depends on the chosen time point $t_{0} \in[0, T)$. The fixed points of the Poincaré map are $z_{0}=0$ and

$$
z^{*}\left(t_{0}\right)=\frac{e^{r T}-1}{a\left(T+t_{0}, t_{0}\right)} .
$$

The trivial fixed point is unstable and the positive point is globally asymptotically stable for the Poincaré map. The trivial fixed point corresponds to the zero solution of the forced logistic equation 55), whereas the positive point corresponds to a globally asymptotically stable periodic orbit

$$
\gamma\left(t, t_{0}\right)=\frac{z^{*}\left(t_{0}\right) e^{r\left(t-t_{0}\right)}}{1+z^{*}\left(t_{0}\right) a\left(t, t_{0}\right)}
$$

Since we have an explicit formula for the Poincaré map and its positive fixed point, we can explicitly calculate the derivative as

$$
\frac{\mathrm{d}}{\mathrm{d} z} \mathbf{P}\left(z, t_{0}\right)_{\mid z=z^{*}\left(t_{0}\right)}=\mathbf{B}\left(t_{0}\right)=\mathrm{e}^{-r T}
$$

Somewhat surprisingly, this derivative is independent of $t_{0}$. Since the exponent is negative, the derivative is less than unity, which proves that the positive steady state is locally stable. From the linearized discrete equation

$$
\mathbf{w}(n+1)=\mathbf{B}\left(t_{0}\right) \mathbf{w}(n)
$$

We calculate the reactivity according to 40 as

$$
\sigma_{P}=-r T<0
$$

Hence, the positive periodic orbit is not period reactive.

Since an explicit calculation of the Poincaré map is generally not possible, one can alternatively calculate its linearization by solving the linearzed equation of the forced continuous-time equation over one period; see main text. For the periodic logistic equation, this direct calculation can be done analytically as follows. 
The linearized equation is

$$
\frac{\mathrm{d}}{\mathrm{d} t} y=r\left(1-\frac{\gamma\left(t, t_{0}\right)}{K(t)}\right) y
$$

Its solution is explicitly given by

$$
y(t)=y\left(t_{0}\right) \exp \left(\int_{t_{0}}^{t} r-\frac{2 r \gamma\left(s, t_{0}\right)}{K(s)} \mathrm{d} s\right) .
$$

Hence, the solution after one period is

$$
y\left(t_{0}+T\right)=y\left(t_{0}\right) \mathrm{e}^{r T} \exp \left(-\int_{t_{0}}^{t_{0}+T} \frac{2 r \gamma\left(s, t_{0}\right)}{K(s)} \mathrm{d} s\right) .
$$

It turns out that the integral can be calculated explicitly with a little bit of patience. Substituting the explicit expression for the periodic orbit from $(62)$, we find that it equals

$$
2 \int_{t_{0}}^{t_{0}+T} \frac{z^{*}\left(t_{0}\right) r \mathrm{e}^{r\left(s-t_{0}\right)} / K(s)}{1+z^{*}\left(t_{0}\right) r \int_{t_{0}}^{s} \mathrm{e}^{r\left(\tau-t_{0}\right)} / K(\tau) \mathrm{d} \tau} \mathrm{d} s=: 2 \int_{t_{0}}^{t_{0}+T} \frac{f^{\prime}(s)}{1+f(s)} \mathrm{d} s .
$$

Direct integration gives

$$
2 \ln \left(\frac{1+f\left(t_{0}+T\right)}{1+f\left(t_{0}\right)}\right)=2 \ln \left(1+f\left(t_{0}+T\right)\right)
$$

since $f\left(t_{0}\right)=0$. When we substitute the explicit expression for the periodic orbit and the fixed point $z^{*}\left(t_{0}\right)$, we find that the expression simplifies to $2 r T$. Hence, we get

$$
y\left(t_{0}+T\right)=y\left(t_{0}\right) \mathrm{e}^{r T} \mathrm{e}^{-2 r T}=y\left(t_{0}\right) \mathrm{e}^{-r T},
$$

which agrees with the derivative found above.

\section{References}

Caswell, H., Neubert, M., 2005. Reactivity and transient dynamics of discrete-time ecological systems. Journal of Difference Equations and Applications 11, 295-310.

Hastings, A., Abbott, K., Cuddington, K., Francis, T., Gellner, G., Lai, Y.C., Morozov, A., Petrovskii, S., Scranton, K., Zeeman, M., 2018. Transient phenomena in ecology. Science 361, eaat6412. 
Hastings, A., Powell, T., 1991. Chaos in a three-species food chain. Ecology 72, 896-903.

Kot, M., 2001. Elements of Mathematical Ecology. Cambridge University Press, Cambridge.

415 Krebs, C., Boutin, S., Boonstra, R. (Eds.), 2001. Ecosystem Dynamics of the Boreal Forest: The Kluane Project. Oxford University Press, Oxford; New York.

Leslie, P., 1948. Some further notes on the use of matrices in population mathematics. Biometrika $35,213-245$.

Mailleret, L., Lemesle, V., 2009. A note on semi-discrete modeling in the life sciences. Philosophical Transactions of the Royal Society London Series A 367, 4779-4799.

Murray, J.D., 2002. Mathematical Biology II: Spatial Models and Biomedical Applications. Springer-Verlag, Berlin.

Neubert, M., Caswell, H., 1997. Alternatives to resilience for measuring the responses of ecological systems to perturbations. Ecology 78, 653-665.

Neubert, M., Caswell, H., Murray, J., 2002. Transient dynamics and pattern formation: reactivity is necessary for Turing instabilities. Mathematical Biosciences 175, 1-11.

Perko, L., 2000. Differential equations and dynamical systems. Springer, New York.

Pimm, S., Lawton, J., 1977. Number of trophic levels in ecological communities. Nature 268, 329.

Turchin, P., 2003. Complex Population Dynamics. volume 35 of Monographs in Population Biology. Princeton University Press, Princeton, NJ.

Vesipa, R., Ridolfi, L., 2017. Impact of seasonal forcing on reactive ecological systems. Journal of Theoretical Biology 419, 23-35.

Wang, X., Lutscher, F., 2018. Turing patterns in a predator-prey model with seasonality. Journal of Mathematical Biology 78, 711-737.

Wang, X., Lutscher, F., 2019. How spatial heterogeneity affects transient behavior in reactiondiffusion systems for ecological interactions. Bulletin of Mathematical Biology 81, 3889-3917. 